International Atomic Energy Agency

and

United Nations Educational Scientific and Cultural Organization

INTERNATIONAL CENTRE FOR THEORETICAL PHYSICS

\title{
DENSITY-DEPENDENT PHONORITON STATES IN HIGHLY EXCITED SEMICONDUCTORS
}

\author{
Nguyen Hong Quang] \\ International Centre for Theoretical Physics, Trieste, Italy. \\ Nguyen Minh Khue \\ Institute of Theoretical Physics, P.O.Box 429 Bo Ho, Hanoi 10000, Vietnam
}

\begin{abstract}
The dynamical aspects of the phonoriton state in highly-photoexcited semiconductors is studied theoretically. The effect of the exciton-exciton interaction and nonbosonic character of high-density excitons are taken into account. Using Green's function method and within the Random Phase Approximation it is shown that the phonoriton dispersion and damping are very sensitive to the exciton density, characterizing the excitation degree of semiconductors.
\end{abstract}

PACS numbers: $63.20 \mathrm{~L}, 71.35,71.36,78.20 \mathrm{~W}$

Keywords: exciton, polariton, phonoriton, nonlinear interaction, high excitation

$$
\text { MIRAMARE - TRIESTE }
$$

August 1995

\footnotetext{
${ }^{1}$ On leave of absence from: Institute of Theoretical Physics, P.O. Box 429, Hanoi 10000, Vietnam.
} 


\section{INTRODUCTION}

It is expected that under an intense electromagnetic radiation near the exciton resonance, the interaction between excitons and phonons can lead to the formation of a new kind of elementary excitation in semiconductors called phonoriton [1-13]. In treating the problem all the authors in [1-13] have considered excitons as ideal bosons and in some cases, have neglected the exciton-exciton interaction, although as mentioned therein, dealing with phonoriton problem, in fact we have matter with high-excitation phenomena, where these assumptions are no more valid. It is well known that in highly-excited semiconductors the so-called giant polariton states are drastically different from that of the usual Hopfield polariton. In such a condition when the number of excitons is very large, the excitons cannot be considered as ideal bosons, and the interaction between them becomes considerable and cannot be neglected. The nonbosonic character of excitons and the exciton-exciton interaction can lead to many interesting effects in semiconductors [14-16]. As a mixture of excitons, photons and phonons, the phonoriton spectrum and damping must depend on the degree of excitation. However, up to now there has been no papers concerning this aspect of the phonoriton problem.

The purpose of our work is to develop an approach to study the dynamical aspects of the phonoriton reconstruction in highly-excited semiconductors. For simplicity, we shall use the simple two-level model recently developed by Wang and Birman [11] to consider both the above-mentioned effects on the phonoriton state. As will be seen the phonoriton spectrum and damping are very sensitive to the exciton density characterizing the excitation degree of semiconductors.

We use the unit system with $\hbar=c=1$.

\section{THE EFFECTIVE HAMILTONIAN}

Let us consider a semiconductor with direct band gap illuminated by high-intensity pump field resonant to the lowest $1 \mathrm{~S}$ exciton state. In such condition the occupation number for the exciton in mode $k$ with frequency equal to that of the incident field, will be very large (mode $k$ is macroscopically occupied). The creation and annihilation operators of the exciton $a_{k}^{+}, a_{k}$ satisfy the following exact commutation relations [16]

$$
\begin{aligned}
{\left[a_{k}, a_{k^{\prime}}\right] } & =\left[a_{k}^{+}, a_{k^{\prime}}^{+}\right]=0, \\
{\left[a_{k}, a_{k^{\prime}}^{+}\right] } & =\delta_{k, k^{\prime}}-\frac{1}{V} \sum_{p}\left\{\varphi^{*}(p-\beta k) \varphi\left(p-\beta k^{\prime}\right) e_{k^{\prime}-p}^{+} e_{k-p}+\right.
\end{aligned}
$$




$$
\left.+\varphi^{*}(p+\alpha k) \varphi\left(p+\alpha k^{\prime}\right) h_{k^{\prime}+p}^{+} h_{k+p}\right\}
$$

where $e_{p}^{+}\left(e_{p}\right), h_{p}^{+}\left(h_{p}\right)$ are the creation (annihilation) operators for the electron and hole with momentum $p$, respectively. $\varphi(p)$ is the Fourier transform of the exciton envelope function for electron-hole relative motion. $\alpha=m_{e} /\left(m_{e}+m_{h}\right)=1-\beta, m_{e}$ and $m_{h}$ are the effective masses of the electron and hole, respectively. $V$ is the volume of the crystal. From (1) it is clear that the exciton can be considered as ideal boson only at low excitation, when the second and third terms on the right-hand side of (1) are neglected.

In addition to the Hamiltonian (1) in Ref.[11] we must also include the term describing the nonlinear exciton-exciton interaction. So our initial Hamiltonian has the following form:

$$
\begin{aligned}
H & =H^{e x-\gamma}+H^{e x-p h} \\
H^{e x-\gamma} & =\omega_{k}^{e x} a_{k}^{+} a_{k}+\omega_{k}^{\gamma} b_{k}^{+} b_{k}+g_{k}\left(a_{k}^{+} b_{k}+b_{k}^{+} a_{k}\right)+f_{k} a_{k}^{+} a_{k}^{+} a_{k} a_{k} \\
H^{e x-p h} & =\sum_{p} \Omega_{p} c_{p}^{+} c_{p}+\sum_{p, q} i M(p-q) a_{p}^{+} a_{q}\left(c_{p-q}-c_{q-p}^{+}\right)
\end{aligned}
$$

where $a_{k}^{+}\left(a_{k}\right), b_{k}^{+}\left(b_{k}\right), c_{k}^{+}\left(c_{k}\right)$ are creation (annihilation) operators for nonbosonic excitons, photons and phonons, respectively; $\omega_{k}^{e x}$ is the energy of the exciton with momentum $k ; \omega_{k}^{e x}=E_{t}+k^{2} / 2 m_{e x}\left(E_{t}\right.$ is the exciton energy at $k=0, m_{e x}$ is the effective mass of exciton), $\omega_{k}^{\gamma}$ is the energy of the photon,$\Omega_{p}$ is the energy of the optical phonon, $g_{k}$ is coefficient of exciton-photon interaction, $M(p-q)$ is the matrix element of exciton-phonon interaction, $f_{k}$ denotes the constant of exciton-exciton interaction. Note that unlike [11] $a_{k}^{+}$and $a_{k}$ stand here for nonbosonic excitons and their commutators satisfy (1). Due to this nonbosonity of the excitons $H^{e x-\gamma}$ in (2) can not be diagonalized by the Bogoliubov transformation like it was done in [11-13]. To overcome this difficulty we introduce the following Green's functions

$$
\begin{aligned}
& G_{1}(k, t)=\theta(t)<\left[a_{k}(t), a_{k}^{+}(o)\right]>\equiv<<a_{k}(t), a_{k}^{+}(o)>>, \\
& G_{2}(k, t) \equiv<<b_{k}(t), a_{k}^{+}(o)>>
\end{aligned}
$$

where the symbol $\langle\ldots\rangle$ denotes the thermodynamical average.

The equations of motion for the Green's functions (5) can be derived in standard way. In order to make the obtained system of equation closed we use RPA-type factorization [17] which seems to be reasonable for high density systems where the fluctuation of the exciton densities can be neglected. As a result we obtain the closed system of equation 
for Green's functions in energy representation

$$
\begin{aligned}
{\left[\omega_{k}^{p o l}-\omega_{k}^{e x}\left(1-\rho_{k}\right)-2 f_{k} n_{e x}\left(1-\rho_{k}\right)\right] G_{1}(k)-g_{k}\left(1-\rho_{k}\right) G_{2}(k) } & =\frac{1}{2 \pi}\left(1-\rho_{k}\right), \\
g_{k} G_{1}(k)-\left(\omega_{k}^{p o l}-\omega_{k}^{\gamma}\right) G_{2}(k) & =0,
\end{aligned}
$$

where $n_{e x}$ is the exciton density in the semiconductors, and

$$
\rho_{k}=\sum_{p}\left\{|\varphi(p-\beta k)|^{2} n_{e}(k-p)+|\varphi(-p+\alpha k)|^{2} n_{h}(k-p)\right\}
$$

$n_{e}(k), n_{h}(k)$ are the electron and hole densities, respectively.

Solving (6) we obtain the dispersion of the polariton i.e. dressed exciton as a pole of the Green's function in the following form

$$
\begin{aligned}
\omega_{i, k}^{p o l} & =\frac{1}{2}\left[\left(\omega_{k}^{e x}+2 n_{e x} f_{k}\right)\left(1-\rho_{k}\right)+\omega_{k}^{\gamma}\right]- \\
& -\frac{(-1)^{i}}{2}\left\{\left[\left(\omega_{k}^{e x}+2 n_{e x} f_{k}\right)\left(1-\rho_{k}\right)-\omega_{k}^{\gamma}\right]^{2}+4\left(1-\rho_{k}\right) g_{k}^{2}\right\}^{\frac{1}{2}},
\end{aligned}
$$

$i=1,2$.

In order to study the phonoriton state it is convenient for us to work with bosons rather than nonbosonic particles. This idea can be realized if we construct the following effective Hamiltonian for the exciton-photon part in term of boson-excitons $\tilde{a}_{k}$ so that this Hamiltonian gives us the same polariton dispersion (8)

$$
H_{e f f}^{e x-\gamma}=\tilde{\omega}_{k}^{e x} \tilde{a}_{k}^{+} \tilde{a}_{k}+\omega_{k}^{\gamma} b_{k}^{+} b_{k}+\tilde{g}_{k}\left(\tilde{a}_{k}^{+} b_{k}+b_{k}^{+} \tilde{a}_{k}\right)
$$

Indeed, if we chose $\tilde{\omega}_{k}^{e x}$ and $\tilde{g}_{k}$, as follows

$$
\begin{aligned}
\tilde{\omega}_{k}^{e x} & =\left(\omega_{k}^{e x}+f_{k} 2 n_{e x}\right)\left(1-\rho_{k}\right), \\
\tilde{g}_{k} & =g_{k} \sqrt{1-\rho_{k}},
\end{aligned}
$$

we can diagonalize the effective Hamiltonian (9) by a canonical Bogoliubov transformation to get it in the form

$$
H_{e f f}^{e x-\gamma}=\sum_{k, i} \omega_{i, k}^{p o l} B_{i k}^{+} B_{i k}
$$

where $\omega_{i, k}^{p o l}$ is exactly defined by $(8) . B_{i k}^{+}$is the creation operator for the polariton in i-branch and with momentum $k$, and expressed in terms of photon $\left(b_{k}\right)$ and boson-exciton $\left(\tilde{a}_{k}\right)$, as follows

$$
B_{i k}=u_{i}(k) b_{k}+v_{i}(k) \tilde{a}_{k},
$$


where

$$
\begin{aligned}
u_{i}(k) & =\frac{\tilde{g}_{k}}{\omega_{i k}^{p o l}-\omega_{k}^{\gamma}} v_{i}(k), \\
v_{i}(k) & =\frac{\left|\omega_{i k}^{p o l}-\omega_{k}^{\gamma}\right|}{\left\{\left(\omega_{i k}^{p o l}-\omega_{k}^{\gamma}\right)^{2}+\tilde{g}_{k}^{2}\right\}^{1 / 2}} .
\end{aligned}
$$

Substituting (11) and (12) into (2), and following the same arguments as in [11] we obtain the final effective Hamiltonian for the anti-Stokes scattering of the incident macroscopically filled polariton mode $k_{o}$ of lower polariton branch.

$$
\begin{aligned}
H_{A S} & =\sum_{p}\left(\omega_{p}^{p o l}+i \gamma_{p}^{p o l}\right) B_{p}^{+} B_{p}+\sum_{p}\left(\Omega_{p}+i \gamma_{p}^{p h}\right) c_{p}^{+} c_{p}+ \\
& +\sum_{p \neq k_{o}} i \tilde{M}\left(p-k_{o}\right)\left(B_{p}^{+} B_{k_{o}} c_{p-k_{o}}-B_{k_{o}}^{+} B_{p} c_{p-k_{o}}^{+}\right)
\end{aligned}
$$

where

$$
\begin{aligned}
\omega_{p}^{p o l} & \equiv \omega_{2, p}^{p o l} \\
\tilde{M}\left(p-k_{o}\right) & \equiv M\left(p-k_{o}\right) v_{2}^{*}(p) v_{2}\left(k_{o}\right) .
\end{aligned}
$$

Note that the Hamiltonian (14) formally looks like Hamiltonian (12) in [11], however,

there is an essential difference between them. While the dispersion of the polariton $\omega_{p}^{p o l}$ in [11] is fixed in respect to external excitation, our $\omega_{p}^{p o l}$ is function of exciton density characterizing the degree of excitation. This is a dynamical consequence of the nonlinear exciton-exciton interaction and the nonbosonity of high density excitons. Moreover, we have also included lifetime effects introducing $\gamma_{p}^{p o l}$ and $\gamma_{p}^{p h}$ - the damping of polariton and phonon, respectively.

\section{PHONORITON SPECTRUM AND DAMPING}

In this section we shall use the effective Hamiltonian derived in the previous section to study phonoriton states in highly excited semiconductors.

Starting now from (14) one can follow [11] replacing $B_{k_{o}}, B_{k_{o}}^{+}$by their c-number expectation values $\left\langle B_{k_{o}}>\right.$ and $\left\langle B_{k_{o}}^{+}>\right.$to get phonoriton dispersion. However, as shown in our previous work [13] such a semiclassical approximation may omit many interesting properties of phonoritons. So in this paper we shall use the Green's function method [17], which is more reasonable and consistent for us in the framework of the RPA. Thus, let us consider the following Green's functions

$$
F_{1}(p, t)=<<B_{p}(t), B_{p}^{+}(0)>>
$$




$$
F_{2}(p, t)=<<B_{k_{o}}(t) c_{p-k_{o}}(t), B_{p}^{+}(0)>>.
$$

The equations of motion for the Green's functions (16) can be derived in standard way. It is easy to check that in the framework of the RPA the Fourier images of the Green's functions (16) are the solutions of the following closed system of equations

$$
\begin{aligned}
\left(\omega-\omega_{p}^{p o l}-i \gamma_{p}^{p o l}\right) F_{1}(p, \omega)-i \tilde{M}\left(p-k_{o}\right) F_{2}(p, \omega) & =\frac{1}{2 \pi}, \\
i \tilde{M}\left(p-k_{o}\right) n_{e x} V F_{1}(p, \omega)+\left(\omega-\omega_{k_{o}}^{p o l}-i \gamma_{k_{o}}^{p o l}-\Omega_{p-k_{o}}-i \gamma_{p-k_{o}}^{p h}\right) F_{2}(p, \omega) & =0,
\end{aligned}
$$

where we have approximately replaced the occupation number $N_{k_{o}}=<B_{k_{o}}^{+} B_{k_{o}}>$ of the polariton at mode $k_{o}$ by $N_{e x}=V \cdot n_{e x}$ due to the exciton-like nature of the polariton mode $k_{o}$ near the exciton resonance.

From the solutions of the system of equations (17) we extract the poles of the functions $F_{1}(p, \omega)$ and $F_{2}(p, \omega)$, the real and imaginary parts of which, as usual, give us the spectrum and damping of the new quasiparticle - the phonoriton.

For the spectrum of phonoriton we obtain the following final expression

$$
\begin{aligned}
W_{i}(p) & =\frac{1}{2}\left(\omega_{p}^{p o l}+\omega_{k_{o}}^{p o l}+\Omega_{p-k_{o}}\right)+ \\
& +(-1)^{i+1} \frac{1}{2}\left\{\frac{1}{2}\left[D(p)^{2}+4 \tilde{M}^{2}\left(p-k_{o}\right) V n_{e x}-\gamma^{2}(p)\right]+\right. \\
& \left.+\frac{1}{2} \sqrt{\left[D(p)^{2}+4 \tilde{M}^{2}\left(p-k_{o}\right) V n_{e x}-\gamma^{2}(p)\right]^{2}+4 D(p)^{2} \gamma^{2}(p)}\right\}^{\frac{1}{2}},
\end{aligned}
$$

and for the damping of phonoriton we have

$$
\begin{aligned}
\Gamma_{i}(p) & =\frac{1}{2} \Gamma(p)+(-1)^{i+1} \frac{1}{2} D(p) \gamma(p)\left\{\frac{1}{2}\left[D(p)^{2}+4 \tilde{M}^{2}\left(p-k_{o}\right) V n_{e x}-\gamma^{2}(p)\right]+\right. \\
& \left.+\frac{1}{2} \sqrt{\left[D(p)^{2}+4 \tilde{M}^{2}\left(p-k_{o}\right) V n_{e x}-\gamma^{2}(p)\right]^{2}+4 D(p)^{2} \gamma^{2}(p)}\right\}^{-\frac{1}{2}}
\end{aligned}
$$

where

$$
\begin{gathered}
D(p)=\omega_{p}^{p o l}-\omega_{k_{o}}^{p o l}-\Omega_{p-k_{o}}, \\
\gamma(p)=\gamma_{p}^{p o l}-\gamma_{p-k_{o}}^{p h}, \\
\Gamma(p)=\gamma_{p}^{p o l}+\gamma_{p-k_{o}}^{p h}, \\
\omega_{p}^{p o l}=\frac{1}{2}\left[\left(\omega_{k}^{e x}+2 f_{k} n_{e x}\right)\left(1-\rho_{k}\right)+\omega_{k}^{\gamma}\right]- \\
-\frac{1}{2}\left\{\left[\left(\omega_{k}^{e x}+2 f_{k} n_{e x}\right)\left(1-\rho_{k}\right)-\omega_{k}^{\gamma}\right]^{2}+4 g_{k}^{2}\left(1-\rho_{k}\right)\right\}^{\frac{1}{2}} .
\end{gathered}
$$


$\rho_{k} \approx \lambda \pi \cdot a_{B}^{3} \cdot n_{e x}[16]$, where $\lambda$ is some constant parameter. The formulas (18) and (19) are the final expressions for the dispersion and damping of the phonoriton. As we see, these analytical expressions depend explicitly on the exciton density that characterises the level of excitation. From (18) we have a new gap of phonoriton

$$
\begin{aligned}
\Delta(p) & =\left\{\frac{1}{2}\left[D(p)^{2}+4 \tilde{M}^{2}\left(p-k_{o}\right) V n_{e x}-\gamma^{2}(p)\right]+\right. \\
& \left.+\frac{1}{2} \sqrt{\left[D(p)^{2}+4 \tilde{M}^{2}\left(p-k_{o}\right) V n_{e x}-\gamma^{2}(p)\right]^{2}+4 D(p)^{2} \gamma^{2}(p)}\right\}^{\frac{1}{2}}
\end{aligned}
$$

It is clear from (18)-(19) that the phonoriton dispersion and damping depend on exciton density not only via $4 \tilde{M}^{2}\left(p-k_{o}\right) V n_{e x}$ as in [10-12], but also through the polariton dispersion (23). If we put $f_{k}=0$ and $\rho_{k}=0$ we reobtain the results of [11].

To see the sensibility of phonoriton states to the exciton density, in Fig.1, 2, and 3 we have plotted the phonoriton dispersion, its gap and damping in $\mathrm{CdS}$ for some exciton densities, respectively. The damping of polaritons can be calculated via the damping of excitons and photon (see for example [18]), and $f=13 \pi E_{B} a_{B}^{3} / 3 V$ [19], where $a_{B}$ is the effective Bohr radius of the exciton and $E_{B}$ is its binding energy. The data used for numerical calculation are adopted from [11]: $E_{t}=2553 \mathrm{mev}, g_{o}=50 \mathrm{mev}, a_{B}=$ $2.8 \times 10^{-7} \mathrm{~cm}, E_{B}=27.8 \mathrm{mev}, k_{o}=3.98 \cdot 10^{5} \mathrm{~cm}^{-1}, m_{e x}=0.98 m_{o}, \gamma^{e x}=0.1 \mathrm{meV}, \gamma^{p h}=$ $0.05 \mathrm{meV}$. Note that the higher exciton density the lower two phonoriton branches go down. With increasing exciton density not only the phonoriton gap becomes larger as in [11], but also the phonoriton resonance region shifts to higher values of momentum. This situation may affect experimental detection of phonoriton and must be taken into account.

\section{CONCLUSION}

In this paper we study for the first time the dynamical aspects of the phonoriton states in highly excited semiconductors. Unlike the previous papers on phonoritons considering the polariton as fixed in respect to external excitation before it comes to interaction with phonon, we have shown that the polariton itself depends on excitation. The intrinsic nonlinear interactions to high-density systems such as nonlinear exciton-exciton interaction and nonbosonic nature of excitons are taken into account. As consequence, these nonlinear interactions lead to dynamical reconstruction of phonoriton states. At fixed $k_{o}$-mode of photon excitation, the higher exciton density (the higher excitation degree) the deeper the phonoriton resonance region goes down, and the larger values of momentum $k$ the latter shifts to. Note that if we neglect these interactions, i.e. $f_{k} \rightarrow 0$ and $\rho_{k} \rightarrow 0$ all 
our results return to the previous ones of [11]. The sensibility of the phonoriton spectrum and damping upon the degree of external excitation usually forgotten up to now should be taken into account, especially in experimental detection of phonoritons. And we hope that our specific predictions will stimulate further experiments.

\section{Acknowledgements}

One of us (N.H.Q) would like to take this opportunity to express his gratitude to the International Centre for Theoretical Physics, Trieste, for hospitality. He would also like to thank Prof. B.F. Zhu for a reading of the manuscript and discussions, and Prof. Yu $\mathrm{Lu}$ for encouragements. 


\section{REFERENCES}

1. A.L. Ivanov and L.V. Keldysh, Dokl. Acad. Nauk.USSR, 264, 1363 (1982)

2. A.L. Ivanov and L.V. Keldysh, Zh. Eksp. Teor. Fiz. 84, 404 (1983)

3. A.L. Ivanov, Zh. Eksp. Teor. Fiz. 90, 158 (1986)

4. L.V. Keldysh and S.G. Tikhodeev, Zh. Eksp. Teor. Fiz. 90, 1852 (1986)

5. L.V. Keldysh and S.G. Tikhodeev, Zh. Eksp. Teor. Fiz. 91, 78 (1986)

6. G.S. Vyskoviskii, G.P. Golubev, E.A. Zhukov, A.A. Fomichev, and M.A. Yaskin, Pis'ma Zh. Eksp. Teor. Fiz. 42, 134 (1985)

7. M.S. Brodin, V.N. Kadan and M.G. Matsko, Fiz. Tver. Tela 30, 1265 (1988)

8. B.I. Greene, J.F. Mueller, J. Orenstein, D.H. Rapkine, S. Schmitt-Rink, and M. Thakar, Phys. Rev. Lett. 61, 325 (1988)

9. T. Ishihara, J. Phys. Soc. Jpn. 57, 2573 (1988)

10. M.I. Schmigliuk and V.N. Pitei, Coherent polaritons in semiconductors. Schtiintsa edt., Kishinev, 1989

11. B.S. Wang and J.L. Birman, Solid State Commun. 75, 867 (1990); Phys. Rev. B 42, 9609 (1990).

12. N.A. Viet, N.Q. Huong, and L.Q. Thong, ICTP's Preprint IC/92/320 (1992)

13. N.M. Khue, N.Q. Huong and N.H. Quang, J.Phys.: Condens. Matter 6, 3221 (1994)

14. M.L. Steyn-Ross and C.W. Gardiner, Phys. Rev. A 27, 310 (1983)

15. N.B. An, Intern. J. Mod. Phys. B5, 1215 (1991)

16. S.A. Moskalenko, Introduction to theory of high density excitons. Schtiintsa edt., Kishinev, 1983

17. A.A. Abrikosov, L.P. Gorkov and I.E. Dzyaloshinski Methods of quantum field theory in statistical physics. Prentice-Hall, Inc., Englewood Cliffs, Newjersey 1963

18. H.N. Cam, N.V. Hieu and N.A. Viet, Phys. stat. sol.(b) 126, 247 (1984)

19. T. Hiroshima, Phys. Rev. B 40, 3862 (1989); J. Phys.: Condens. Matter, 4, 3849 (1992) 


\section{FIGURE CAPTIONS}

Fig.1 The dispersion of the anti-Stokes phonoriton at some exciton densities $n_{e x}=1 \cdot 10^{17}$ $\mathrm{cm}^{-3}$ (thick line), $1.5 \cdot 10^{17} \mathrm{~cm}^{-3}$ (thin line), $2 \cdot 10^{17} \mathrm{~cm}^{-3}$ (dotted line).

Fig.2 The function $\Delta(p)$ at some exciton densities $n_{e x}=1 \cdot 10^{17} \mathrm{~cm}^{-3}$ (thick line), $1.5 \cdot 10^{17}$ $\mathrm{cm}^{-3}$ (thin line), $2 \cdot 10^{17} \mathrm{~cm}^{-3}$ (dotted line).

Fig.1 The damping of the anti-Stokes phonoriton at some exciton densities $n_{e x}=1 \cdot 10^{17}$ $\mathrm{cm}^{-3}$ (thick line), $1.5 \cdot 10^{17} \mathrm{~cm}^{-3}$ (thin line), $2 \cdot 10^{17} \mathrm{~cm}^{-3}$ (dotted line). 